\title{
Identification of long non-coding RNAs biomarkers for early diagnosis of myocardial infarction from the dysregulated coding- non-coding co-expression network
}

\author{
Chaoyu Sun ${ }^{1}$, Hao Jiang ${ }^{2}$, Zhiguo Sun ${ }^{2}$, Yifang Gui ${ }^{3}$, Hongyuan Xia ${ }^{1}$ \\ ${ }^{1}$ Department of Cardiology, The Fourth Affiliated Hospital of Harbin Medical University, Harbin 150001, China \\ ${ }^{2}$ Department of General Surgery, The Affiliated Hongqi Hospital of Mudanjiang Medical University, Mudanjiang 157011, China \\ ${ }^{3}$ The Clinical Laboratory, The Affiliated Hongqi Hospital of Mudanjiang Medical University, Mudanjiang 157011, China \\ Correspondence to: Hongyuan Xia, email: hongyxia@yeah.net
}

Keywords: biomarkers, diagnosis, long non-coding RNAs, myocardial infarction

Received: July 06, $2016 \quad$ Accepted: August 24, 2016

Published: September 13, 2016

\section{ABSTRACT}

Long non-coding RNAs (IncRNAs) have recently been shown as novel promising diagnostic or prognostic biomarkers for various cancers. However, IncRNA expression patterns and their predictive value in early diagnosis of myocardial infarction (MI) have not been systematically investigated. In our study, we performed a comprehensive analysis of IncRNA expression profiles in MI and found altered IncRNA expression pattern in MI compared to healthy samples. We then constructed a IncRNA-mRNA dysregulation network (DLMCEN) by integrating aberrant IncRNAs, mRNAs and their co-dysregulation relationships, and found that some of mRNAs were previously reported to be involved in cardiovascular disease, suggesting the functional roles of dysregulated IncRNAs in the pathogenesis of MI. Therefore, using support vector machine (SVM) and leave one out cross-validation (LOOCV), we developed a 9-IncRNA signature (termed 9LncSigAMI) from the discovery cohort which could distinguish MI patients from healthy samples with accuracy of $95.96 \%$, sensitivity of $93.88 \%$ and specificity of $\mathbf{9 8 \%}$, and validated its predictive power in early diagnosis of MI in another completely independent cohort. Functional analysis demonstrated that these nine IncRNA biomarkers in the 9LncSigAMI may be involved in myocardial innate immune and inflammatory response, and their deregulation may lead to the dysfunction of the inflammatory and immune system contributing to MI recurrence. With prospective validation, the 9LncSigAMI identified by our work will provide additional diagnostic information beyond other known clinical parameters, and increase the understanding of the molecular mechanism underlying the pathogenesis of MI.

\section{INTRODUCTION}

Myocardial Infarction (MI), commonly known as heart attack, is a serious result of coronary artery disease (CAD) caused by sudden blockage or extremely reduced blood flow in a coronary artery. MI remains the major cause of death and mortality globally, including China [1]. Over the past years, there is a sharply increasing trend in the morbidity of MI in China. Although statistics in 2011 suggested that there were about two million cases of MI accounting for $0.87 \%$ of cardiovascular disease (CVD)[2], it is estimated by that the number of patients with MI will increase to 23 million by 2030 [3]. Early diagnosis identifying subpopulations at high risk of having an infarct is crucial for deciding early tailored treatment to reduce MI mortality. Although currently available biomarkers, such as cardiac troponin and creatine kinaseMB (CK-MB), have used to assist with timely diagnosis $[4,5]$, some novel molecular biomarkers have highlighted their promising potentials and important roles for early management in MI. 
Recent genome sequencing and transcriptomics analyses have revealed that only less than two percent of the human genome consists of protein-coding RNA, whereas the majority of the genome can be transcribed into RNA transcripts without protein coding capacity [6]. These non-coding RNAs (ncRNAs) can be classified into two types based on their size: short RNAs and long non-coding RNAs (lncRNAs). Short RNAs, including microRNAs (miRNAs), have been widely studied during the past ten years. Many studies have reported the dysregulation of miRNA expression in MI, including $m i R-15$ [7], $m i R-21$ [8], miR-24 [9], $m i R-29$ [10] and so on. LncRNAs, a major class of ncRNAs, was defined as ncRNA transcript with greater than 200 nucleotides. Since the lncRNAs H19 and Xist were firstly found to be involved in epigenetic regulation in the early 1990s [11, 12], increasing evidence has shown that IncRNAs are involved in the complex gene regulation network by as key molecular players at epigenetic, transcriptional and post-transcriptional levels [13, 14]. A handful of studies have revealed dysregulated expression of IncRNAs in a variety of disease states highlighting their potential clinical application as diagnostic and prognostic biomarkers or therapeutic targets in the pathology of diverse diseases, including cancers [15-17]. Some studies have reported several lncRNA-focus signatures which could be useful to predict patients' prognosis or metastasis in various cancers [18-28]. Recent studies have shown the close association between MI and altered lncRNA expression [29, 30], highlighting the potential of lncRNAs as biomarkers in early diagnosis of MI. However, IncRNA expression patterns and their predictive value for MI have not been systematically investigated.

In this study, we obtained lncRNA expression profiles and investigated the expression patterns between MI patients and healthy samples by re-annotating the publicly available Affymetrix microarray. Furthermore, we constructed and analyzed a dysregulated lncRNAmRNA co-expression network that is associated with the occurrence of MI. We aimed to detect potential lncRNA biomarkers closely correlated with MI, and to develop novel lncRNA signature for identifying subpopulations at high risk of having an infarct

\section{RESULTS}

\section{Identification of deregulated mRNA and IncRNA in AMI patients}

We first performed SAM analysis to investigate the expression patterns of mRNAs and IncRNAs between AMI patients and healthy samples from the discovery cohort and identified 191 and 11 differentially expressed mRNAs and $\operatorname{lncRNAs}(\log \mathrm{FC} \leq 1$ or $\log \mathrm{FC}>1$, FDR-adjusted $\mathrm{p}$ $<0.05)$. Among them, 175 mRNAs and 9 lncRNAs were over-expressed and 16 mRNAs and 2 lncRNAs were down-expressed in AMI patients compared with healthy samples (Supplementary Table S1).

\section{Construction and analysis of dysregulated IncRNA-mRNA co-expression network}

We first investigated the co-expression correlations between differentially expressed mRNAs and differentially expressed lncRNAs. The lncRNA-mRNA pairs with a high PCC $(>0.5)$ were selected as significantly dysregulated IncRNA-mRNA co-expression pairs and were integrated into the DLMCEN, in which there are 1822 edges between 188 mRNAs and 11 lncRNAs (Figure 1A). The investigation of the degree distribution of nodes in DLMCEN revealed a power-law distribution with a slope of -1.674 and $\mathrm{R}^{2}=0.999$ (Figure 1B), suggesting that DLMCEN has scale-free characteristics like many other biological networks distinguished from random networks. Moreover, the degree and betweenness centrality of lncRNAs are significantly higher than those of mRNAs (Wilcoxon rank-sum test) (Figure 1C and 1D), demonstrating that dysregulated lncRNAs tended to be hub nodes and played more important roles than mRNAs in the occurrence of AMI.

\section{Construction of SVM-based IncRNA signature in AMI diagnosis from the discovery cohort}

To identify an optimal lncRNA signature predictive of AMI, we have searched for lncRNA combinations among the 11 dysregulated lncRNAs in the DLMCEN, whose expression pattern can best distinguish AMI patients from the control samples, using SVM and LOOCV strategy in the discovery cohort. As shown in Figure 2A, a signature of 9 lncRNAs (termed 9LncSigAMI) with the highest accuracy was identified (Table 1). The classification of 99 samples in the discovery cohort using the 9LncSigAMI classifier achieved an accuracy of $95.96 \%$ with a sensitivity of $93.88 \%$ and a specificity of $98 \%$ (Figure 2B). The discriminatory power measured by AUC is 0.985 (Figure 2B). We also applied hierarchical clustering analysis to expression data of nine lncRNAs in the 9LncSigAMI from 49 AMI patients and 50 healthy samples and found 2 major sample clusters with clear differences in lncRNA expression patterns. As shown in Figure 2C, all healthy samples were grouped into Cluster 2 and most of AMI patients (33/49, 67.35\%) were grouped into Cluster 1, revealing a significant association between lncRNA expression pattern and samples' disease status $(\mathrm{p}=5.431 \mathrm{e}-12$, Chi-square test; Figure 2C). The above results demonstrated the good performance of the 9LncSigAMI in distinguishing AMI patients from healthy samples in the discovery cohort. Of these nine diagnostic biomarkers, eight lncRNAs tended to be risky 
lncRNAs whose up-regulated expression associated with AMI occurrence and only one lncRNAs was protective lncRNAs whose down-regulated expression associated with AMI occurrence (Figure 2C and 2D).

\section{Validation of the 9LncSigAMI with an additional independent cohort}

To evaluate the robustness of the 9LncSigAMI, we conducted a further validation of the predictive power of 9LncSigAMI using an additional independent cohort of 52 samples from Suresh's study [31] (denoted "validation cohort"). We first performed a hierarchical clustering analysis based on the expression pattern of these nine diagnostic biomarkers. 52 samples in the validation cohort were clearly clustered into two distinct subgroups (Figure $3 \mathrm{~A})$, with significantly different disease status $(\mathrm{p}=2.27 \mathrm{e}-$
02, Chi-square test). As observed in the discovery cohort, eight protective lncRNAs showed a higher expression in healthy samples in Cluster 1 and one risky lncRNA showed a higher expression in AMI samples in Cluster 2.

We further assessed the predictive power of the 9LncSigAMI using SVM algorithm and LOOCV procedure. On the validation cohort, the 9LncSigAMI for distinguishing AMI patients from healthy samples achieves an AUC of 0.771 with a sensitivity of $70.97 \%$ and a specificity of $61.91 \%$ (Figure $3 \mathrm{~B}$ ). The 9LncSigAMI correctly classified 22 out of 31 AMI samples and 13 out of 21 control samples, resulting in $67.31 \%$ prediction accuracy. These results suggested that the 9LncSigAMI signature identified here was accurate and reliable for discriminating AMI patients from normal samples.

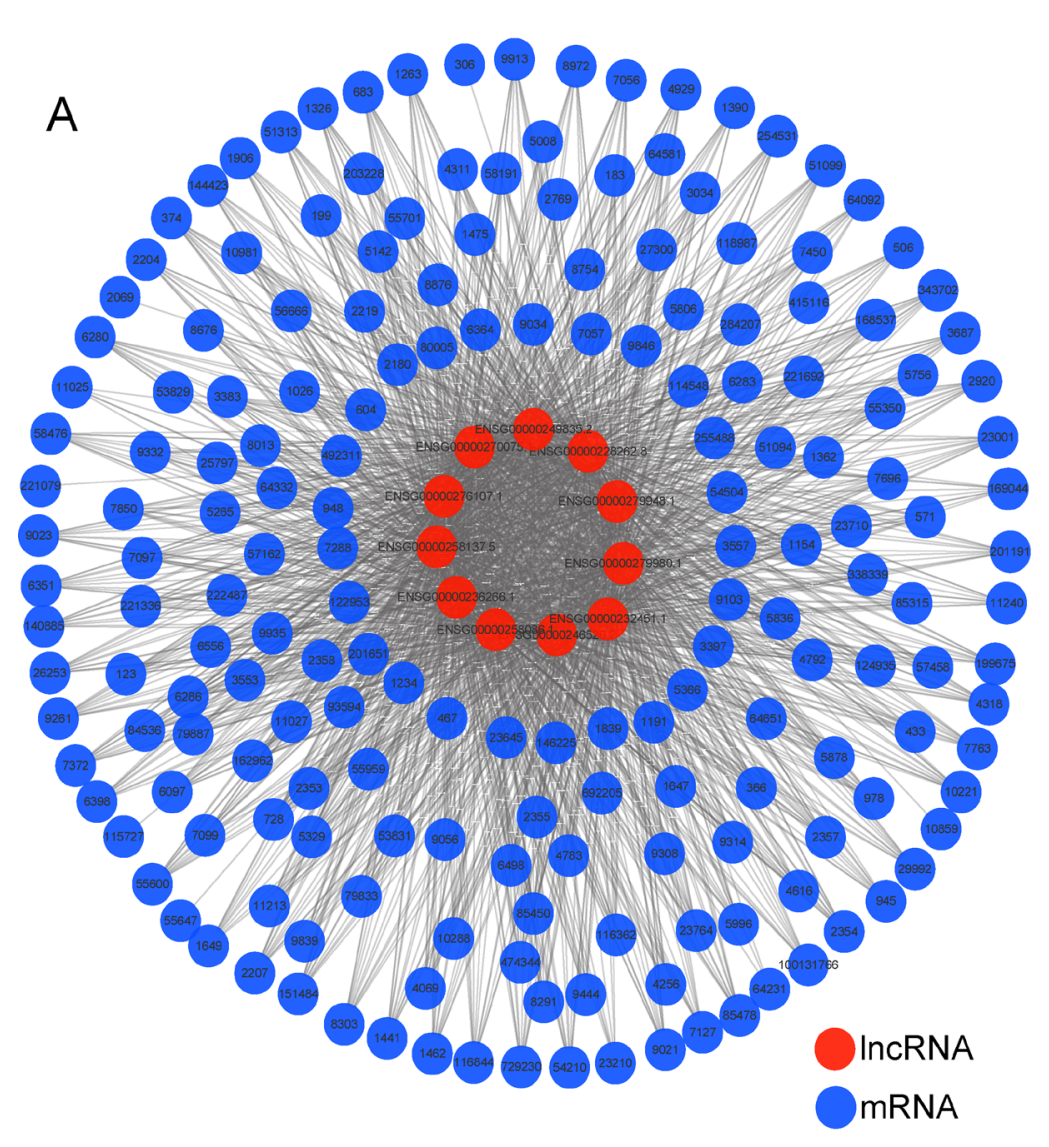

B

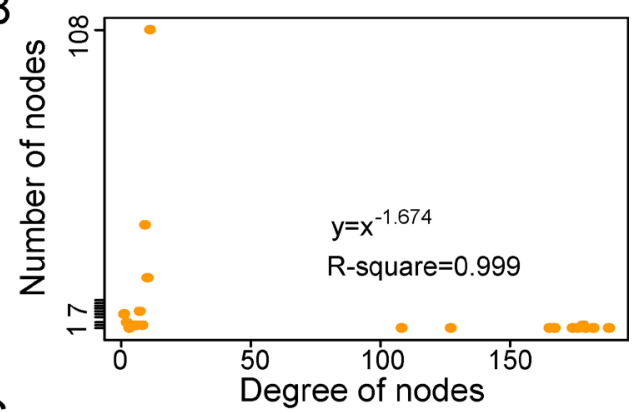

C

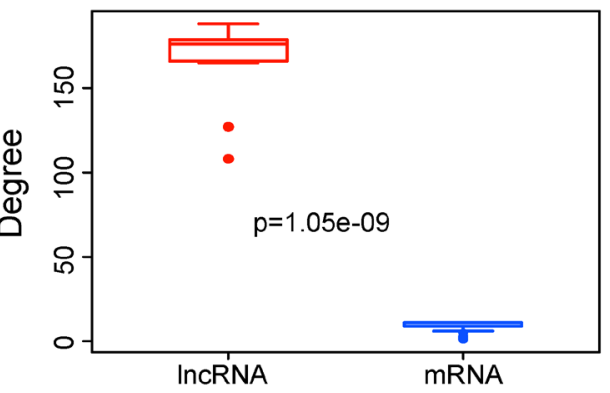

D

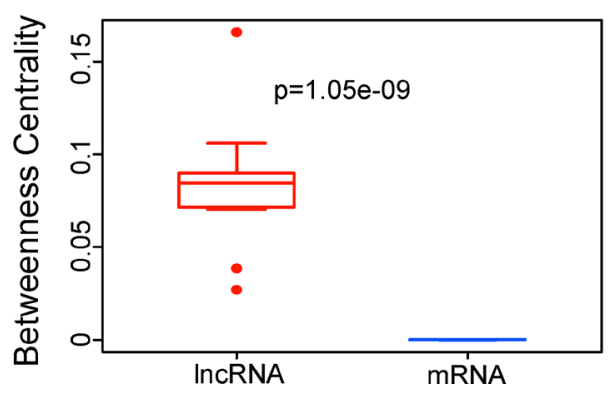

Figure 1: Construction and analysis of MI-related dysregulated IncRNA-mRNA co-expression network. A. The DLMCEN generated by the procedure described in the Methods, including 1822 edges between 188 mRNAs and 11 lncRNAs. B. Degree distribution of the DLMCEN. C. The LncRNAs have significantly higher degrees than mRNAs in the DLMCEN. D. The lncRNAs have significantly higher betweenness centrality than mRNAs the DLMCEN. 


\section{Identification of associated biological pathways of the 9LncSigAMI}

As an initial step to infer potential biological functions of the 9LncSigAMI, we first examined the expression correlation between mRNAs and nine lncRNAs using the Pearson correlation coefficient and identified 517 mRNAs positively correlated with at least one of the nine lncRNAs. Then GO and KEGG function enrichment analysis for mRNAs co-expressed with lncRNAs was conducted to identify associated biological processes and pathways of the 9LncSigAMI. Results with GO analysis revealed that mRNAs coexpressed with nine lncRNAs tended to be significantly enriched in 30 GO terms in the "Biological Process" (GOTERM-BP-FAT) (adjusted p-value $<0.10$ and an enrichment score of $>1.5$ ) (Supplementary Table S2), which were grouped into four functional clusters including immune response, inflammatory response, regulation of cytokine and cell death (Figure 4A). The
A

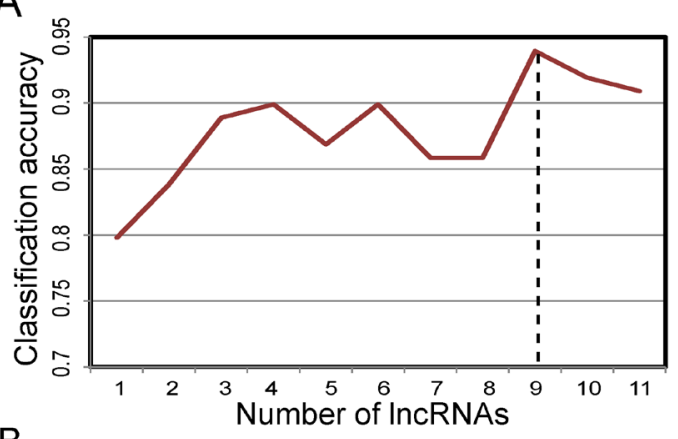

B

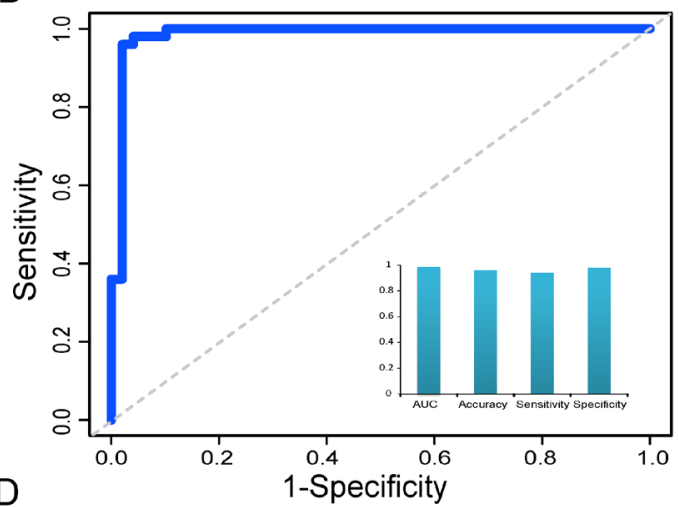

D

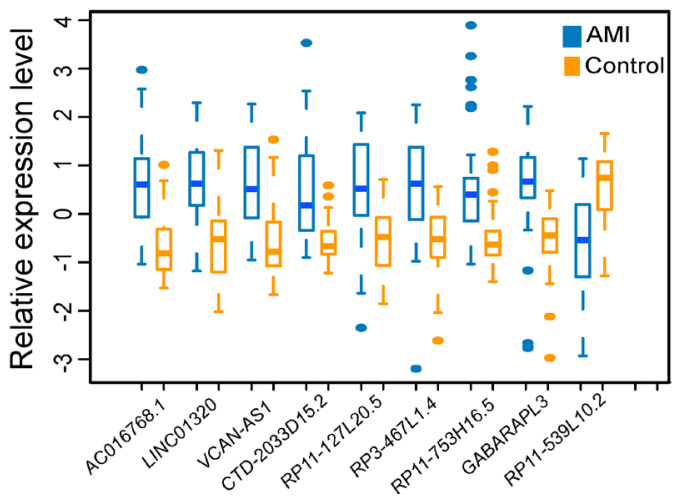

C

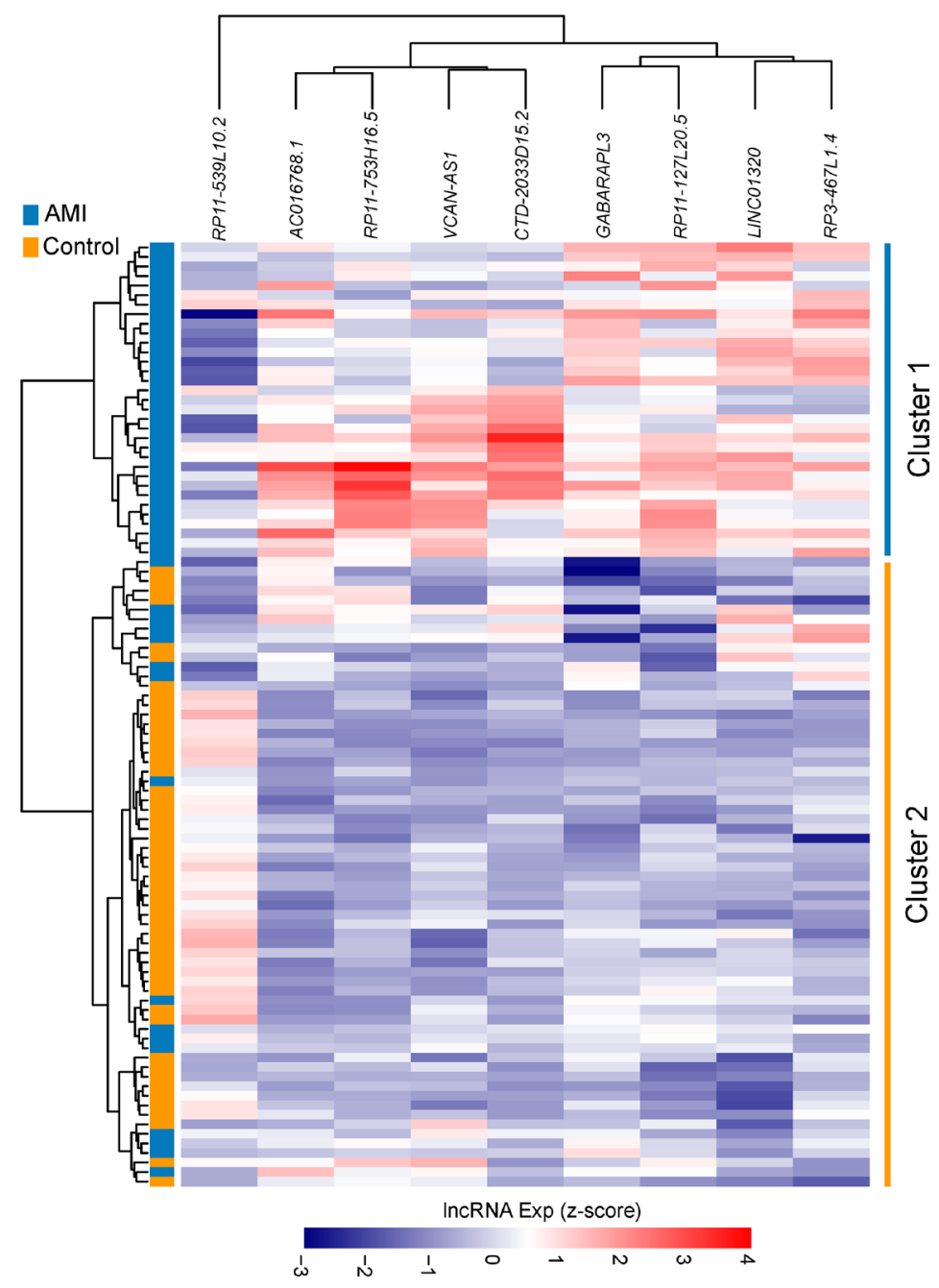

Figure 2: Identification and predictive value of SVM-based IncRNA signature in AMI diagnosis from the discovery cohort. A. Classification performance of different numbers of lncRNA biomarkers. B. Performance evaluation of the 9LncSigAMI in early diagnosis using LOOCV procedure in the discovery cohort. C. The hierarchical clustering heat map of 99 samples based on expression profiles of 9 lncRNAs in the 9LncSigAMI in the discovery dataset. D. The expression levels of 9 lncRNAs in the 9LncSigAMI between MI patients and healthy samples 
Table 1: The detailed information of IncRNA biomarkers in the 9LncSigAMI

\begin{tabular}{lccc}
\hline LncRNA ID & Ensembl name & Genome locations & $\operatorname{logFC}$ \\
\hline ENSG00000246526 & RP11-539L10.2 & Chr 4: 6,687,448-6,690,519(-) & -1.02 \\
ENSG00000232451 & AC016768.1 & Chr 2: 23,018,125-23,199,056(-) & 1.30 \\
ENSG00000258086 & RP11-753H16.5 & Chr 12: 54,353,792-54,466,985(+) & 1.08 \\
ENSG00000249835 & VCAN-AS1 & Chr 5: 83,531,352-83,581,320(-) & 1.26 \\
ENSG00000276107 & CTD-2033D15.2 & Chr 15: 39,586,561-39,587,293(+) & 1.15 \\
ENSG00000279980 & GABARAPL3 & Chr 15: 90,347,587-90,349,437(+) & 1.04 \\
ENSG00000270075 & RP11-127L20.5 & Chr 10: 104,312,141-104,313,881(+) & 1.11 \\
ENSG00000228262 & LINC01320 & Chr 2: 34,677,555-34,738,231(+) & 1.27 \\
ENSG00000236266 & RP3-467L1.4 & Chr 1: 7,810,242-7,827,342(-) & 1.08 \\
\hline
\end{tabular}

analysis of KEGG showed that mRNAs co-expressed with nine lncRNAs were involved in several pathways including Hematopoietic cell lineage, Cytokine-cytokine receptor interaction, Adipocytokine signaling pathway, Toll-like receptor signaling pathway, Chemokine signaling pathway and NOD-like receptor signaling pathway (Supplementary Table S3). These biological processes and pathways have been reported to be close with myocardial infarction [32-39], suggesting that the perturbation of nine lncRNAs in the 9LncSigAMI played important parts in the occurrence of AMI by interacting with mRNAs involved in known MI-related biological processes and pathways.

\section{DISCUSSION}

Increasing evidence has revealed that lncRNA has complex and important roles in cardiovascular diseases, including MI [40, 41]. For example, the down-regulated expression of IncRNA UCA1 has been observed at the early state of AMI patients [29]. Vausort and colleagues measured expression levels of five lncRNAs in 414 patients using quantitative PCR, and identified a lncRNA MIAT highly relevant to MI [30]. The above studies have demonstrated the potential roles of lncRNAs as promising novel biomarkers for the diagnosis and/or prognosis of MI. However, the current research strategies focused on molecular diagnostic or prognostic biomarkers have mainly analyzed expression profiles of mRNA or miRNAs [5] [42, 43]. Although transcriptome analysis has been reported in the AMI mouse model [44], there is a lack of the investigation into expression patterns and diagnostic values of IncRNA in MI patients owing to the limitation of available expression data in human. Recent studies revealed that lncRNA expression profiles could be obtained by re-annotating the probes in the commonly used microarrays [18, 19, 23, 45].

In this study, we obtained and analyzed lncRNA expression profiles of 151 samples (80 AMI patients and 71 healthy samples) by repurposing two publicly available microarray expression datasets to determine whether there is significantly altered lncRNA expression pattern between AMI patients and healthy samples. We observed significantly different lncRNA expression patterns in AMI patients compared to healthy samples and identified 11 differentially expressed lncRNAs, implying that these dysregulated lncRNAs may be associated with MI. Previous studies have demonstrated that IncRNAs function as key regulators of gene expression by interacting with protein-coding genes to participate in biological processes [46, 47]. Therefore, to identify functional lncRNAs and its partners, we investigated the co-expression relationship between dysregulated lncRNAs and dysregulated mRNAs and constructed a dysregulated IncRNA-mRNA coexpression network (DLMCEN). As general biological networks, the DLMCEN exhibited scale-free and modular characteristics. These dysregulated lncRNAs were found to be topologically central within DLMCEN and have maximal informational connections with other dysregulated mRNAs. After a comprehensive searching in both genetic association database (GAD) and Online Mendelian Inheritance in Man database (OMIM), 58 protein-coding genes in the DLMCEN have been found to be associated with cardiovascular disease, 18 of which were involved in MI (Supplementary Table S4). These observations indicated that the altered expression of these 11 lncRNAs in the DLMCEN 

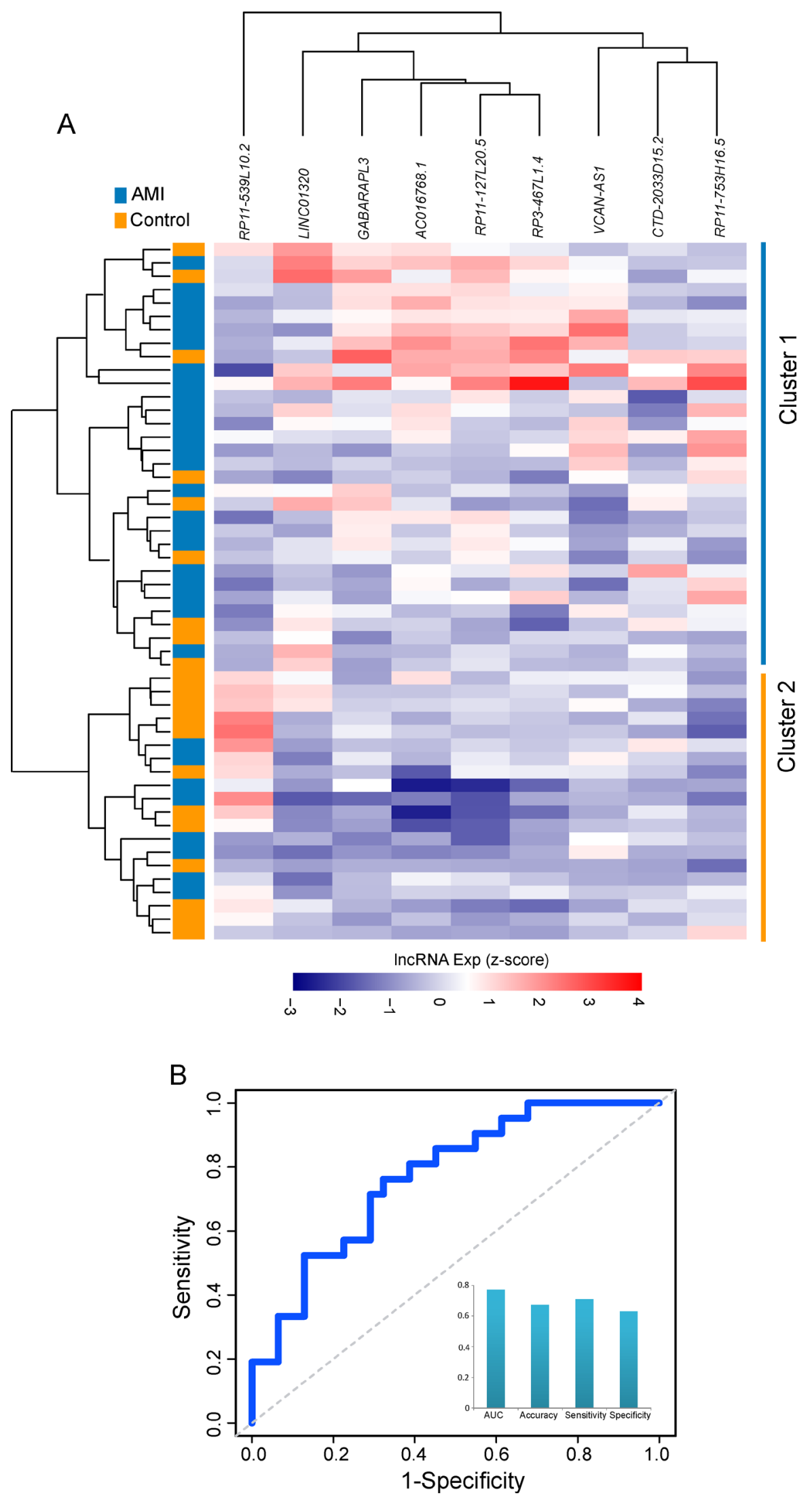

Figure 3: Validation of the 9LncSigAMI in the additional independent cohort. A. The hierarchical clustering heat map of 52 samples based on expression profiles of 9 lncRNAs in the 9LncSigAMI in the test cohort. B. Performance evaluation of the 9LncSigAMI in early diagnosis using LOOCV procedure in the test cohort. 
contributed to the development of MI and could be used as potential biomarkers for early diagnosis of MI patients. Hierarchical clustering analysis revealed that these dysregulated IncRNAs in the DLMCEN were significantly correlated with disease status, highlighting their potential clinically application to assess the risk of MI and improve diagnosis prediction of MI. To identifying an optimal lncRNA signature considering a balance between accuracy and the number of lncRNAs, we used SVM and random forest method to detect a combination of lncRNAs that has a remarkable ability to distinguish AMI patients from healthy samples. After the feature selection procedure, 9 of 11 dysregulated lncRNAs was identified as optimal lncRNAs biomarkers with the highest accuracy. Thus, we developed an SVMbased diagnostic predictor as a lncRNA signature based on expression profiles of nine lncRNAs. The superior performance of the 9LncSigAMI in early AMI detection was further validated in the discovery cohort and another completely independent cohort.

Since only a few of lncRNAs have been annotated functionally, we performed bioinformatics analysis to infer possible associated biological roles of MI-related lncRNAs. From the co-expression network based on dysregulated lncRNAs and mRNAs, we performed functional enrichment analysis for co-expressed mRNAs and found that these mRNAs were enriched in several known MI-related biological processes and pathways. For example, TLR4, the members of the Toll-like receptors (TLR) family in the Toll-like receptor signaling pathway, have been found to play a detrimental role in myocardial ischemia/reperfusion (I/R) injury [48]. Cytokines, an inflammatory factor, were observed to be significantly up-regulated or down-regulated expression in AMI patients, and the altered cytokine expression had impacts on the immune functions in patients with AMI [33]. Chemokines, a family of chemotactic cytokines, acted as a modulator in complex biological processes such as cell proliferation and gene transcription, and its overexpression may be a reparative response following $\mathrm{MI}$ [37]. Therefore, it is a plausible inference that these nine lncRNA biomarkers may be involved in myocardial innate immune and inflammatory response, and their deregulation may lead to the dysfunction of the inflammatory and immune system contributing to MI recurrence.

In summary, we performed a comprehensive survey of the expression profiles of lncRNAs and coding RNAs from MI patients and healthy samples in a large of samples and constructed a dysregulated lncRNAmRNA co-expression network which will improve our understanding of MI-related lncRNAs from a network view. Furthermore, we developed and validated a SVMbased lncRNA signature in use for early diagnosis of AMI with high accuracy. To our knowledge, this study is the first to access the application of lncRNAs for diagnostic prediction of MI. With prospective validation, the lncRNA signature identified by our work will provide additional diagnostic information beyond other known clinical parameters, and increase the understanding of the molecular mechanism underlying the pathogenesis of MI.
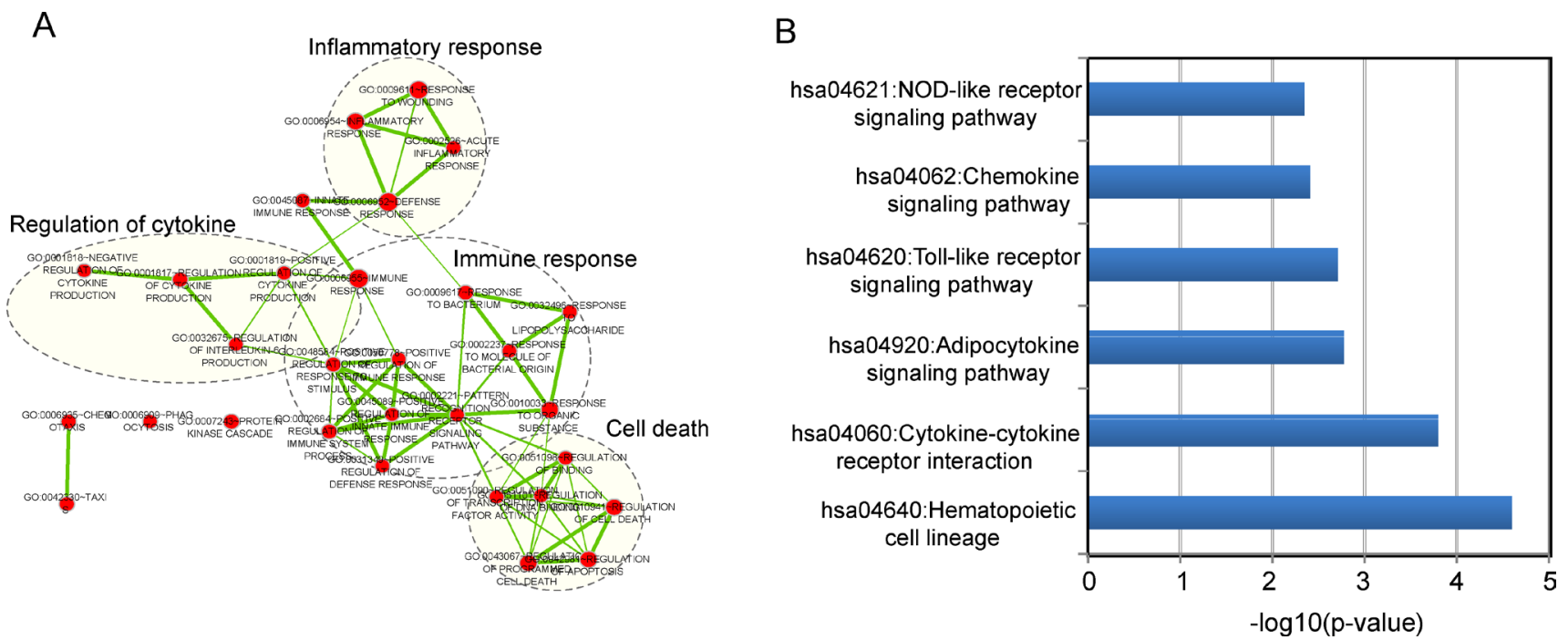

Figure 4: Function enrichment maps of the diagnostic IncRNAs. A. The functional enrichment map of GO terms with each node represents a GO term and an edge represents the proportion of shared genes between connecting GO terms. B. The enriched KEGG pathways ranked by $-\log 10$ (p-value). 


\section{MATERIALS AND METHODS}

\section{Gene expression profile dataset}

The gene expression profile data based on Affymetrix Human Genome U133 Plus 2.0 Array (HGU133_Plus_2.0) from two independent nonoverlapping cohorts of AMI patients were obtained from the publicly available GEO database (www.ncbi.nlm.nih. gov/geo/). The initial discovery cohort of 49 AMI patients and 50 healthy samples were obtained from GEO (GSE66360, http://www.ncbi.nlm.nih.gov/geo/ query/acc.cgi?acc $=$ GSE66360) and used to identify novel lncRNAs biomarkers for AMI diagnosis. Another AMI patient dataset was also downloaded from GEO (GSE48060, http://www.ncbi.nlm.nih.gov/geo/query/acc. cgi?acc $=$ GSE48060)[31], denoted "validation cohort", including 31 AMI patients and 21 healthy samples and was considered as an independent test cohort to validate the diagnostic power of IncRNA biomarkers.

\section{Acquisition of IncRNA expression profiles}

The raw expression profile data (.CEL files) of 99 samples in the discovery cohort and 52 samples in the validation cohort were downloaded from the GEO database. The probe set sequences of Affymetrix HGU133_Plus_2.0 were obtained from the Affymetrix website (http://www.affymetrix.com). LncRNA expression data of 151 samples were obtained using the probe reannotation strategy as previously described $[18,19,24]$. Briefly, probe sets of HG-U133_Plus_2.0 array were aligned to the human genome (GRCh38) and lncRNA gene sequence from GENCODE (release 23) using SeqMap tool with no mismatch [49]. Then lncRNAspecific probes were obtained by mapping the genomic locations of probes to the genomic locations of lncRNAs. Finally, expression data of 2332 lncRNA were obtained for further analysis.

\section{Expression profiles analysis}

The significance analysis of microarrays (SAM) method was used to identify differentially expressed lncRNAs and mRNAs between AMI patients and healthy samples. The expression variation from AMI patients to healthy samples was characterized by $\log \mathrm{FC}(\log 2$ fold change) and associated p-values adjusted after BenjaminiHochberg false discovery rate (FDR) control approach [50]. Down- and up-regulated mRNAs and lncRNAs were selected with the cut-off criterion of a $\operatorname{logFC}<$ -1 and $\log \mathrm{FC}>1$ respectively, with FDR-adjusted $\mathrm{p}<$ 0.05 . Hierarchical clustering analysis was carried out to investigate the patterns of IncRNA expression in the different samples, and the chi-square test was used to analyze the correlations between AMI status and lncRNA biomarkers.

\section{Construction and analysis of dysregulated IncRNA-mRNA co-expression network}

The dysregulated lncRNA-mRNA co-expression network (DLMCEN) in AMI patients was constructed as follows: Firstly, Pearson correlation coefficient (PCC) was calculated by measuring the expression relationships between differentially expressed mRNAs and differentially expressed lncRNAs. Then IncRNA-mRNA pairs with a high PCC $(>0.5)$ were selected as dysregulated lncRNAmRNA co-expression pairs. Finally, a DLMCEN was constructed for AMI by assembling all dysregulated lncRNA-mRNA co-expression pairs identified above. A node represents a lncRNA or mRNA, and mRNA and IncRNA are connected if they are differentially coexpressed.

\section{Construction of SVM-based IncRNA signature in AMI diagnosis}

A lncRNA-focus predictive signature for sample classification was developed using the support vector machine (SVM) with the sigmoid kernel. The performance of SVM-based lncRNA signature was estimated using the leave one out cross-validation (LOOCV). Sensitivity, specificity and accuracy were calculated through a $2 \times$ 2 contingency table, and the ROC curve was drawn by plotting true positive rates (sensitivity) against false positive rates (1-specificity).

To construct an optimal lncRNA signature in AMI diagnosis, optimal lncRNA biomarkers were selected using the random forest supervised classification algorithm as follows: (i) candidate lncRNA biomarkers were ranked according to their random forest importance value. (ii) The SVM-based signature was developed by adding one lncRNA at a time in a top-down order starting with the first two lncRNAs in the lncRNA ranking list, and the performance of the SVM-based signature was evaluated using LOOCV. (iii) The optimal number of IncRNA biomarkers in the signature could be found when achieving the highest classification accuracy.

\section{Functional analysis of IncRNA biomarkers}

The expression correlations between lncRNA biomarkers and mRNAs were calculated using the Pearson correlation coefficients (PCCs). The mRNAs positively correlated with biomarkers ( $\mathrm{PCCs}>0.60$ ) was chosen as co-expressed mRNAs associated with lncRNA signature. We performed bioinformatics analysis to predict the function of IncRNA signature by functional enrichment analysis of Gene Ontology (GO) and Kyoto encyclopedia of genes and genomes (KEGG) for coexpressed mRNAs. Functional enrichment analysis was carried out using DAVID Bioinformatics Tool (version 6.7) which is widely used to discover the biological implications of a set of genes [51]. Enriched GO terms 
limited in "Biological Process" (GOTERM-BP-FAT) and KEGG pathways with an adjusted p-value of $<0.10$ using the Benjamini-Hochberg procedure and an enrichment score of $>1.5$ were considered as significant functional annotations. Enrichment maps of significant GO terms were constructed and visualized using the Enrichment Map plugin in Cytoscape software [52].

\section{CONFLICTS OF INTEREST}

The authors declare that they have no of interest.

\section{ACKNOWLEDGMENTS}

This work was supported by the Natural Science Foundation of Heilongjiang Province of China (Grant No. H201387)

\section{REFERENCES}

1. Maracy MR, Isfahani MT, Kelishadi R, Ghasemian A, Sharifi F, Shabani R, Djalalinia S, Majidi S, Ansari H, Asayesh H, Qorbani M. Burden of ischemic heart diseases in Iran, 1990-2010: Findings from the Global Burden of Disease study 2010. J Res Med Sci. 2015; 20:1077-1083.

2. Li H, Ge J. Cardiovascular diseases in China: Current status and future perspectives. IJC Heart \& Vasculature. 2015; 6:25-31.

3. Li J, Li X, Hu S, Yu Y, Yan XF, Jiang LX. [ST-segment elevation myocardial infarction in the eastern urban China: from 2001 to 2011]. Zhonghua Xin Xue Guan Bing Za Zhi. 2016; 44:303-308.

4. Zhang L, Chen X, Su T, Li H, Huang Q, Wu D, Yang C, Han Z. Circulating miR-499 are novel and sensitive biomarker of acute myocardial infarction. J Thorac Dis. 2015; 7:303-308.

5. Chan D, Ng LL. Biomarkers in acute myocardial infarction. BMC Med. 2010; 8:34.

6. Consortium EP, Birney E, Stamatoyannopoulos JA, Dutta A, Guigo R, Gingeras TR, Margulies EH, Weng Z, Snyder M, Dermitzakis ET, Thurman RE, Kuehn MS, Taylor CM, et al. Identification and analysis of functional elements in $1 \%$ of the human genome by the ENCODE pilot project. Nature. 2007; 447:799-816.

7. Hullinger TG, Montgomery RL, Seto AG, Dickinson BA, Semus HM, Lynch JM, Dalby CM, Robinson K, Stack C, Latimer PA, Hare JM, Olson EN, van Rooij E. Inhibition of miR-15 protects against cardiac ischemic injury. Circ Res. 2012; 110:71-81.

8. Roy S, Khanna S, Hussain SR, Biswas S, Azad A, Rink C, Gnyawali S, Shilo S, Nuovo GJ, Sen CK. MicroRNA expression in response to murine myocardial infarction: miR-21 regulates fibroblast metalloprotease-2 via phosphatase and tensin homologue. Cardiovasc Res. 2009; 82:21-29.

9. Wang J, Huang W, Xu R, Nie Y, Cao X, Meng J, Xu X, $\mathrm{Hu} \mathrm{S}$, Zheng Z. MicroRNA-24 regulates cardiac fibrosis after myocardial infarction. J Cell Mol Med. 2012; $16: 2150-2160$.

10. van Rooij E, Sutherland LB, Thatcher JE, DiMaio JM, Naseem RH, Marshall WS, Hill JA, Olson EN. Dysregulation of microRNAs after myocardial infarction reveals a role of miR-29 in cardiac fibrosis. Proc Natl Acad Sci U S A. 2008; 105:13027-13032.

11. Brannan CI, Dees EC, Ingram RS, Tilghman SM. The product of the H19 gene may function as an RNA. Molecular and cellular biology. 1990; 10:28-36.

12. Brockdorff N, Ashworth A, Kay GF, McCabe VM, Norris DP, Cooper PJ, Swift S, Rastan S. The product of the mouse Xist gene is a $15 \mathrm{~kb}$ inactive $\mathrm{X}$-specific transcript containing no conserved ORF and located in the nucleus. Cell. 1992; 71:515-526.

13. Kornienko AE, Guenzl PM, Barlow DP, Pauler FM. Gene regulation by the act of long non-coding RNA transcription. BMC Biol. 2013; 11:59.

14. Wang KC, Chang HY. Molecular mechanisms of long noncoding RNAs. Mol Cell. 2011; 43:904-914.

15. Fatima R, Akhade VS, Pal D, Rao SM. Long noncoding RNAs in development and cancer: potential biomarkers and therapeutic targets. Mol Cell Ther. 2015; 3:5.

16. Zhou M, Wang X, Li J, Hao D, Wang Z, Shi H, Han L, Zhou $\mathrm{H}$, Sun J. Prioritizing candidate disease-related long noncoding RNAs by walking on the heterogeneous lncRNA and disease network. Mol Biosyst. 2015; 11:760-769.

17. Sun J, Shi H, Wang Z, Zhang C, Liu L, Wang L, He W, Hao D, Liu S, Zhou M. Inferring novel lncRNA-disease associations based on a random walk model of a lncRNA functional similarity network. Mol Biosyst. 2014; 10:2074-2081.

18. Zhou M, Guo M, He D, Wang X, Cui Y, Yang H, Hao D, Sun J. A potential signature of eight long non-coding RNAs predicts survival in patients with non-small cell lung cancer. J Transl Med. 2015; 13:231.

19. Zhou M, Zhao H, Wang Z, Cheng L, Yang L, Shi H, Yang H, Sun J. Identification and validation of potential prognostic lncRNA biomarkers for predicting survival in patients with multiple myeloma. J Exp Clin Cancer Res. 2015; 34:102.

20. Hu Y, Chen HY, Yu CY, Xu J, Wang JL, Qian J, Zhang X, Fang JY. A long non-coding RNA signature to improve prognosis prediction of colorectal cancer. Oncotarget. 2014; 5:2230-2242. doi: 10.18632/oncotarget.1895.

21. Meng J, Li P, Zhang Q, Yang Z, Fu S. A four-long noncoding RNA signature in predicting breast cancer survival. J Exp Clin Cancer Res. 2014; 33:84.

22. Sun J, Chen X, Wang Z, Guo M, Shi H, Wang X, Cheng L, Zhou M. A potential prognostic long non-coding RNA 
signature to predict metastasis-free survival of breast cancer patients. Sci Rep. 2015; 5:16553.

23. Zhang XQ, Sun S, Lam KF, Kiang KM, Pu JK, Ho AS, Lui WM, Fung CF, Wong TS, Leung GK. A long noncoding RNA signature in glioblastoma multiforme predicts survival. Neurobiol Dis. 2013; 58:123-131.

24. Zhou M, Xu W, Yue X, Zhao H, Wang Z, Shi H, Cheng L, Sun J. Relapse-related long non-coding RNA signature to improve prognosis prediction of lung adenocarcinoma. Oncotarget. 2016; 7:29720-38. doi: 10.18632/oncotarget.8825.

25. Zhou M, Sun Y, Sun Y, Xu W, Zhang Z, Zhao H, Zhong Z, Sun J. Comprehensive analysis of lncRNA expression profiles reveals a novel lncRNA signature to discriminate nonequivalent outcomes in patients with ovarian cancer. Oncotarget. 2016; 7:32433-48. doi: 10.18632/oncotarget.8653.

26. Zhou M, Wang X, Shi H, Cheng L, Wang Z, Zhao H, Yang L, Sun J. Characterization of long non-coding RNAassociated ceRNA network to reveal potential prognostic lncRNA biomarkers in human ovarian cancer. Oncotarget. 2016; 7:12598-12611. doi: 10.18632/oncotarget.7181.

27. Sun J, Cheng L, Shi H, Zhang Z, Zhao H, Wang Z, Zhou M. A potential panel of six-long non-coding RNA signature to improve survival prediction of diffuse large-B-cell lymphoma. Sci Rep. 2016; 6:27842.

28. Zhou M, Diao Z, Yue X, Chen Y, Zhao H, Cheng L, Sun J. Construction and analysis of dysregulated lncRNA-associated ceRNA network identified novel lncRNA biomarkers for early diagnosis of human pancreatic cancer. Oncotarget. 2016; 7:56383-56394. doi: 10.18632/oncotarget.10891.

29. Yan Y, Zhang B, Liu N, Qi C, Xiao Y, Tian X, Li T, Liu B. Circulating Long Noncoding RNA UCA1 as a Novel Biomarker of Acute Myocardial Infarction. Biomed Res Int. 2016; 2016:8079372.

30. Vausort M, Wagner DR, Devaux Y. Long noncoding RNAs in patients with acute myocardial infarction. Circ Res. 2014; 115:668-677.

31. Suresh R, Li X, Chiriac A, Goel K, Terzic A, Perez-Terzic $\mathrm{C}$, Nelson TJ. Transcriptome from circulating cells suggests dysregulated pathways associated with long-term recurrent events following first-time myocardial infarction. J Mol Cell Cardiol. 2014; 74:13-21.

32. Dutta P, Sager HB, Stengel KR, Naxerova K, Courties G, Saez B, Silberstein L, Heidt T, Sebas M, Sun Y, Wojtkiewicz G, Feruglio PF, King K, et al. Myocardial Infarction Activates CCR2 $(+)$ Hematopoietic Stem and Progenitor Cells. Cell Stem Cell. 2015; 16:477-487.

33. Yan W, Wen S, Wang L, Duan Q, Ding L. Comparison of cytokine expressions in acute myocardial infarction and stable angina stages of coronary artery disease. Int J Clin Exp Med. 2015; 8:18082-18089.

34. Sciarretta S, Frati G. The Importance of Restoring the Adiponectin Signaling Pathway to Reduce Myocardial Reperfusion Injury in Diabetes. Diabetes. 2016; 65:826-828.
35. Lu C, Ren D, Wang X, Ha T, Liu L, Lee EJ, Hu J, Kalbfleisch J, Gao X, Kao R, Williams D, Li C. Tolllike receptor 3 plays a role in myocardial infarction and ischemia/reperfusion injury. Biochim Biophys Acta. 2014; 1842:22-31.

36. Feng Y, Chao W. Toll-like receptors and myocardial inflammation. International journal of inflammation. 2011; 2011.

37. Cavalera M, Frangogiannis NG. Targeting the chemokines in cardiac repair. Curr Pharm Des. 2014; 20:1971-1979.

38. Christia P, Frangogiannis NG. Targeting inflammatory pathways in myocardial infarction. Eur J Clin Invest. 2013; 43:986-995.

39. de Haan JJ, Smeets MB, Pasterkamp G, Arslan F. Danger signals in the initiation of the inflammatory response after myocardial infarction. Mediators Inflamm. 2013; 2013:206039.

40. Jiang $X$, Ning $Q$. The emerging roles of long noncoding RNAs in common cardiovascular diseases. Hypertens Res. 2015; 38:375-379.

41. Uchida S, Dimmeler S. Long noncoding RNAs in cardiovascular diseases. Circ Res. 2015; 116:737-750.

42. Wang GK, Zhu JQ, Zhang JT, Li Q, Li Y, He J, Qin YW, Jing Q. Circulating microRNA: a novel potential biomarker for early diagnosis of acute myocardial infarction in humans. Eur Heart J. 2010; 31:659-666.

43. Yin X, Sami M, Hou D, Shahzad A. MicroRNAs (miRNAs) based Biomarkers for Acute Myocardial Infarction (AMI) Diagnosis. Journal of Clinical \& Experimental Cardiology. 2012; 2012.

44. Ounzain S, Micheletti R, Beckmann T, Schroen B, Alexanian M, Pezzuto I, Crippa S, Nemir M, Sarre A, Johnson R, Dauvillier J, Burdet F, Ibberson M, et al. Genome-wide profiling of the cardiac transcriptome after myocardial infarction identifies novel heart-specific long non-coding RNAs. Eur Heart J. 2015; 36:353-368a.

45. Du Z, Fei T, Verhaak RG, Su Z, Zhang Y, Brown M, Chen Y, Liu XS. Integrative genomic analyses reveal clinically relevant long noncoding RNAs in human cancer. Nat Struct Mol Biol. 2013; 20:908-913.

46. Liu Y, Zhang R, Qiu F, Li K, Zhou Y, Shang D, Xu Y. Construction of a lncRNA-PCG bipartite network and identification of cancer-related lncRNAs: a case study in prostate cancer. Mol Biosyst. 2015; 11:384-393.

47. Guo X, Gao L, Liao Q, Xiao H, Ma X, Yang X, Luo H, Zhao G, Bu D, Jiao F, Shao Q, Chen R, Zhao Y. Long non-coding RNAs function annotation: a global prediction method based on bi-colored networks. Nucleic Acids Res. 2013; $41: \mathrm{e} 35$.

48. Vilahur G, Badimon L. Ischemia/reperfusion activates myocardial innate immune response: the key role of the toll-like receptor. Front Physiol. 2014; 5:496. 
49. Jiang H, Wong WH. SeqMap: mapping massive amount of oligonucleotides to the genome. Bioinformatics. 2008; 24:2395-2396.

50. Reiner A, Yekutieli D, Benjamini Y. Identifying differentially expressed genes using false discovery rate controlling procedures. Bioinformatics. 2003; 19:368-375.

51. Huang da W, Sherman BT, Lempicki RA. Bioinformatics enrichment tools: paths toward the comprehensive functional analysis of large gene lists. Nucleic Acids Res. 2009; 37:1-13.

52. Merico D, Isserlin R, Stueker O, Emili A, Bader GD. Enrichment map: a network-based method for gene-set enrichment visualization and interpretation. PLoS One. 2010; 5:e13984. 